\title{
Rasgos de personalidad predictores del Síndrome de Quemarse por el Trabajo en profesores mexicanos
}

\section{Personality Traits that Predict the Burnout Syndrome on Mexican Teachers}

\author{
Diego Villaverde* \\ Universidad Nacional Autónoma de México, Ciudad de México, México \\ ORCID: https://orcid.org/0000-0003-3651-2078 \\ Sara Unda \\ Universidad Nacional Autónoma de México, Ciudad de México, México \\ ORCID: https://orcid.org/0000-0002-6113-055X \\ Eduardo A. Escotto \\ Universidad Nacional Autónoma de México, Ciudad de México, México \\ ORCID: https://orcid.org/0000-0002-1104-8195 \\ Rosa Flores \\ Universidad Nacional Autónoma de México, Ciudad de México, México \\ ORCID: https://orcid.org/0000-0002-0106-5944
}

Recibido 31-05-19 Revisado 19-06-19 Aprobado 13-09-19 En línea 18-09-19

*Correspondencia

Email: villaverdevillaverde95@gmail.com
Citar como:

Villaverde, D., Unda, S., Escotto, E., \& Flores R. (2019). Rasgos de personalidad predictores del Síndrome de Quemarse por el Trabajo en profesores mexicanos. Propósitos y Representaciones, 7(3), 41-71. doi: http://dx.doi.org/10.20511/pyr2019.v7n3.346 


\section{Resumen}

El objetivo fue determinar el valor predictivo de los rasgos de personalidad según el modelo de los Cinco Factores Mexicanos de la Personalidad en el Síndrome de Quemarse por el Trabajo y sus dimensiones desde el modelo del Gil-Monte. Participaron 375 profesores de educación básica de la Ciudad de México, el muestreo fue no probabilístico intencional con diseño transversal y correlacional. Se utilizó el Cuestionario para la Evaluación del Síndrome de Quemarse por el Trabajo y la Escala de los Cinco Factores Mexicanos de Personalidad. Para analizar se utilizó la Prueba de correlación de Pearson, y un modelo de regresión lineal por pasos. A excepción de Control Emocional e Ilusión en Trabajo $\left(\mathrm{r}^{2}=.087 ; p>0.05\right)$, los resultados encontraron correlaciones significativas de los 5FM y el SQT, negativas en el caso de rasgos de personalidad y Desgaste Psíquico, Indolencia, y Culpa, positivas entre 5FM e Ilusión por el Trabajo. El modelo de regresión lineal múltiple incluyó como predictores del SQT a Sociabilidad Expresiva, Organización y Aceptabilidad ( $\left.\mathrm{F}=39.8, \beta=-.197^{*}\right)$. Se concluye que en la población de docentes estos son los rasgos de personalidad asociados al síndrome, pero tienen un papel flexible en el desarrollo del SQT. Los resultados coinciden con otros estudios. Se sugiere para investigaciones posteriores evaluar el de papel modulador de estos rasgos con Factores de Riesgo Psicosocial como antecedente.

Palabras Clave: Estrés laboral; Personalidad; Docente de escuela primaria y secundaria; Reforma de la educación

\section{Summary}

The objective of the study was to determine the predictive value of personality traits according to the Mexican model of the Five Personality Factors in the Burnout Syndrome (BOS) and its dimensions from the Gil-Monte model. Three hundred and seventy-five basic education teachers from Mexico City took part in it. The sampling was intentional, non-probabilistic with crosssectional and correlational design. The Spanish Burnout Syndrome inventory and the Mexican Five Personality Factors scale were used. Pearson's Correlation Test and a stepwise linear regression model were employed for the analysis. With the exception of Emotional Control and Enthusiasm towards work ( $\mathrm{r} 2=.087$; $\mathrm{p}>0.05$ ), the results found significant correlations of the Five Mexican personality factors and BOS, negative in the case of personality traits and Psychic Burnout, Indolence, and Guilt; and positive among personality factors and enthusiasm towards work. The multiple linear regression model included Expressive Sociability, Organization and Acceptableness as BOS predictors $(\mathrm{F}=39.8, \beta=-.197 *)$. Therefore, it is concluded that these are the personality traits associated with the syndrome that have a flexible role in the development of BOS. The results also coincide with other studies. It is suggested for further research to evaluate the modulating role of these traits with Psychosocial Risk Factors as an antecedent.

Keywords: Stress; Personality; Teachers; Educational Reform.

\section{Introducción}

En el trabajo realizado por Gil-Monte (2007) se han identificado en torno al Síndrome de Quemarse por el Trabajo (SQT) diversos factores que tienen un papel en el desarrollo del mismo, señalando variables de índole social, organizacional o personal, dentro de esta última se encuentra a la personalidad, variable identificada como mediadora en el proceso de desarrollo del SQT (ElSahili, 2015). Al respecto Espinoza-Díaz, Tous-Pallarès y Vigil-Colet (2015) señala la importancia de los recursos personales como lo es la personalidad al enfrentar situaciones estresantes.

Algunos análisis y avances sobre el tema se realizaron a finales de la década de los 80 's en países anglosajones tomando como variables de personalidad a la resistencia al cambio, la tendencia a la evitación, la dificultad en la toma de decisiones, el neuroticismo, perfeccionismo e 
idealismo, la extraversión, la planificación y la organización entre otras (El-Sahili, 2015; Llanque, 2014; Ortiz, et al., 2009).

El SQT, es abordado desde diferentes enfoques y evaluado a través de diferentes modelos, el más relevante y señalado en la literatura científica es el aportado por Maslach y Jackson (1981) que lo considera un síndrome tridimensional que se manifiesta en profesionales que atienden a personas y se caracteriza por sentirse agotado emocionalmente, con pérdida de interés por su trabajo y actitudes indolentes y distantes hacia los usuarios del servicio y el instrumento utilizado por excelencia ha sido el Maslach Burnout Inventory (MBI), sin embargo a lo largo de los años se han realizado modificaciones en la manera de evaluar el fenómeno, como el MBI-GS, que está diseñado para su aplicación en poblaciones con cualquier tipo de ocupación.

Maslach y Leiter (2016) refieren diversos esfuerzos para evaluar el SQT que responden a situaciones culturales específicas uno de ellos es el aporte del modelo del Cuestionario para la Evaluación del SQT (Gil-Monte, 2005) que agrega una cuarta dimensión llamada Culpa. Este modelo es de gran relevancia en la población hispanoamericana pues según estudios de Díaz y Gómez, (2016) se ha convertido en una referencia obligada. Por este motivo se toma como el modelo que se utilizará en este estudio.

\section{Modelo de Gil-Monte (Síndrome de Quemarse por el Trabajo)}

Gil-Monte señala que el SQT ha sido definido como un "un síndrome de agotamiento emocional, despersonalización y falta de realización personal en el trabajo que puede desarrollarse en aquellas personas cuyo objeto de trabajo son personas en cualquier tipo de actividad" (Gil-Monte, 2005, p. 45). Se le considera un síndrome caracterizado por deterioro cognitivo y de carácter no psiquiátrico, que comienza con la pérdida del deseo de tener metas en el trabajo y alcanzar estas, o pérdida de Ilusión por el Trabajo como la llama Gil-Monte; este fenómeno es producto de un deterioro emocional que llamado Desgaste Psíquico como lo refiere el mismo modelo. Después están las actitudes y comportamiento de indiferencia, en ocasiones de carácter abusivo hacia el cliente, llamadas Indolencia y por último, la Culpa que es producida por la actitudes o comportamientos indolentes (Figueiredo-Ferraz, Grau-Alberola, \& Gil-Monte, 2016; Gil-Monte, 2007; Rabasa, Figueiredo-Ferraz H, Gil-Monte, \& Llorca-Pellicer, 2016).

Una de las dimensiones que juega un papel importante en este modelo es la culpa, debido a que, en algunas ocasiones, las actitudes de carácter abusivo hacia las personas dentro del trabajo, tanto a compañeros como usuarios del servicio prestado, conllevan altos sentimientos de culpa. Esta idea resulta fundamental para el modelo, la cual consiste en la posibilidad de distinguir dos perfiles diferentes en el proceso de desarrollo del SQT. Por un lado, un perfil que cumple los criterios de alto o crítico en las tres primeras dimensiones del modelo y que no incluye los sentimientos de culpa, se considera entonces que el individuo en esta situación tiene Perfil 1 y por otro, un perfil que además de presentar SQT sí incorpora la culpa (Perfil 2), diferenciando dos tipos de individuos que padecen el síndrome. La culpa por otro lado tiene un contenido simbólico acerca del compromiso con las personas en el entorno de trabajo (Figueiredo-Ferraz, GrauAlberola, \& Gil-Monte, 2016; Gil-Monte, 2007; Rabasa, Figueiredo-Ferraz H, Gil-Monte, \& Llorca-Pellicer, 2016).

Gil-Monte (2007) considera para su modelo cuatro dimensiones que se explican en los profesores de la siguiente forma, la primera de estas es llamada Ilusión por el Trabajo corresponde al deseo del profesor, para alcanzar objetivos en su labor como docente, y este proceso a su vez como fuente de placer personal. Después se encuentra el Desgaste Psíquico, que se ve reflejado en la presencia del agotamiento emocional y físico debido a las condiciones en las que el docente desempeña su trabajo. La Indolencia que es la presencia de actitudes negativas hacia los alumnos, padres de familia y en ocasiones compañeros de trabajo, y la Culpa que en el caso de los profesores es producto de las actitudes antes mencionadas. Pese a que las condiciones laborales en cualquier puesto de trabajo sean más o menos las mismas, cada trabajador responde diferente, 
es decir, su historia personal, sus destrezas psicosociales y sus rasgos de personalidad, acentúan o atenúan el estrés laboral (Gil-Monte, 2007; Rabasa et al., 2016).

A lo largo de los últimos años, diversos estudios han brindado numerosas evidencias sobre la prevalencia del SQT en docentes de todos los niveles del sistema educativo, así como de sus impactos en las instituciones educativas, su productividad y principalmente su salud (Aldrete, Pando, Aranda, \& Balcázar, 2003; Ayuso \& Guillen, 2007; Barbosa, Muñoz, Rueda, \& Suárez, 2009; Silva, García, González, \& Ratto, 2015; Unda, Contreras, Sánchez, \& García, 2008). Lo cual lleva a considerar también factores de índole intrínseco como la personalidad.

\section{Los Cinco Grandes (5G) (Big Five)}

Los $5 \mathrm{G}$ son el esfuerzo de una serie de teóricos quienes propusieron cinco rasgos predominantes y comunes de la personalidad, dentro de estos, se destaca a Raymond Catell (19051998) quien a través del análisis factorial propuso rasgos que permitirían la predicción conductual en algunas situaciones específicas. Fue también a través del esfuerzo de varios investigadores que se concluye con el término acuñado en 1982 de los llamados 5G (Cloninger, 2003; Vicent, Inglés, Gonzálvez, Sanmarin, \& García-Fernández, 2016).

Algunas de las dimensiones de ésta teoría pueden compararse con la teoría de rasgos planteada por Eysenck, es necesario puntualizar que al igual que en la anterior teoría, en los 5G cada rasgo puede ser explicado mediante un eje (Ver Figura 1), en un extremo de este se sitúan los individuos que presentan las predisposiciones de respuesta propias del rasgo, que por ejemplo en la Extroversión son el ser creativo, entusiasta, asertivo, con confianza en sí mismo, además de emociones positivas, calidez y el ser afectivo mientras que aquellos que puntúan bajo no las presentan o presentan niveles bajos estos individuos por consiguiente se encuentran en el extremo opuesto del eje. Esta explicación mediante el eje "Extroversión"- "Baja Extroversión" puede usarse para entender los otros rasgos; "Neuroticismo"- "Bajo Neuroticismo", "Cordialidad""Baja Cordialidad", "Conciencia"- "Baja Conciencia" y "Apertura a la Experiencia"-"Baja Apertura a la Experiencia" (Cloninger, 2003; Vicent et al., 2016).

En esta teoría el Neuroticismo hace referencia a respuestas emocionales, generalmente sentimientos de ansiedad, depresión, ira y descontento, quien recibe una puntuación elevada en este rasgo pueden ser descrito como vulnerable. En este modelo se suman tres rasgos además de los mencionados en lugar del Psicoticismo (de Eysenck) se incluye la Cordialidad o Afabilidad que representa la confianza, la sencillez, el interés por los demás y por sus necesidades, además de la bondad. La Conciencia o Rectitud, que valora la autonomía, precisión, cumplimiento de responsabilidades, orden y organización en general. Y por último la Apertura a la Experiencia, que evalúa aspectos intelectuales, ya sean a través de acciones, ideas, la estética o la fantasía (Cloninger, 2003; Vicent et al., 2016).

Figura 1. Relación entre tradición griega y Teoría de Eysenck

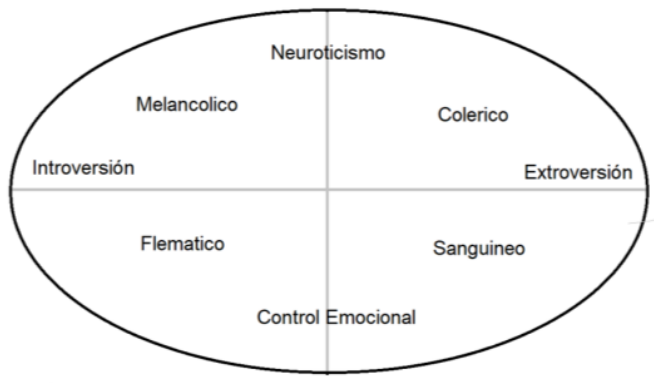

Figura 1. Basada en la relación planteada por Eysenck entre sus planteamientos y la tradición griega. 


\section{Los cinco factores mexicanos de la personalidad (5FM)}

Los 5FM son una adaptación de los 5G (Big Five) a población mexicana realizada por Uribe (Uribe, 2002), proviene de críticas realizadas al uso de instrumentos psicológicos diseñados para culturas ajenas a la mexicana (Uribe \& Arias 2005). En este modelo se consideró el modelo Orientación Ocupacional de Holland (1966) además del modelo de personalidad. Cada una de las siguientes dimensiones representan un eje, en un extremo se sitúan los individuos que prestan el rasgo en menor medida y en el extremo opuesto los que lo presentan en mayor medida (ver Figura 1) (Uribe, 2002; Uribe, Contreras, Sánchez, \& García, 2008):

1. La Sociabilidad Expresiva es entendida como las diferentes formas de expresión que las personas tienen o llevan a cabo al relacionarse con los demás, fluctúa entre introversión y extraversión, y es parte del mismo eje de los 5G. Un nivel bajo de Sociabilidad Expresiva implicaría una mayor introversión y un nivel alto una mayor extroversión (ver Tabla 1).

2. La Aceptabilidad, que corresponde a las características del individuo que determinan si este es o no aceptado por los demás. Si se presenta valores altos es el individuo es mayormente aceptado, ocurre lo contrario si los valores son bajos. Este rasgo puede ser comparado con el rasgo de Cordialidad del modelo de los $5 \mathrm{G}$ ya que corresponde un estilo de interacción con otros individuos.

3. La Organización que corresponde a los estilos o actitudes en la dirección que una persona emplea al realizar actividades o tareas. Se puede compara con la Conciencia en los $5 \mathrm{G}$ ya que la autonomía, la rectitud y la precisión forman parte del estilo de dirección que tiene el individuo al desempeñar una tarea.

4. El Control Emocional consiste en el grado de estabilidad emocional observada en una persona al expresar actos, ideas y pensamientos, este rasgo puede ser comparado con lo que llamamos bajo Neuroticismo en los 5G, ya que el Neocriticismo hace referencia a respuestas emocionales, sentimientos de ansiedad, ira o descontento.

5. Sensibilidad Intelectual que refiere a habilidades o capacidades con las que un individuo expresa y percibe experiencias, vivencias o problemas. Lo podemos comparar con el rasgo de Apertura a la Experiencia explicado en los 5G debido a que en ambos se evalúan aspectos intelectuales del individuo.

El instrumento creado por Uribe (2002) para evaluar los 5FM ha sido validado también en población trabajadora (Uribe \& Arias, 2005; Uribe, Contreras, Sánchez, \& García, 2008), y señalan la necesidad de contar con instrumentos que evalúen aspectos psicológicos como la personalidad y tomen en cuenta condiciones propias de la cultura mexicana con resultados favorables en su comportamiento psicométrico con Alfas entre .72 a .85, por tal motivo, fue el modelo elegido para éste estudio.

La relación que existe entre la personalidad y el SQT ha sido estudiada en algunas poblaciones de trabajadores, aquí se abordan los resultados de alguna de ellas. 


\begin{tabular}{|c|c|c|c|}
\hline Tradición griega & Eysenck & Big Five & $5 \mathrm{FM}$ \\
\hline $\begin{array}{l}\text { Sanguíneo } \\
\text { (extrovertido y } \\
\text { estable). }\end{array}$ & Extroversión & Extroversión & Sociabilidad Expresiva \\
\hline $\begin{array}{l}\text { Flemático (introvertido } \\
\text { y estable). }\end{array}$ & (Baja Extroversión). & (Baja Extroversión). & $\begin{array}{l}\text { (Baja Sociabilidad } \\
\text { Expresiva). }\end{array}$ \\
\hline \multicolumn{4}{|l|}{$\begin{array}{l}\text { Colérico (extroversión } \\
\text { y neuroticismo). }\end{array}$} \\
\hline $\begin{array}{l}\text { Melancólico } \\
\text { (introversión y } \\
\text { neuroticismo) }\end{array}$ & Neuroticismo. & Neuroticismo. & $\begin{array}{l}\text { Control Emocional (o } \\
\text { bajo neuroticismo). }\end{array}$ \\
\hline \multirow[t]{2}{*}{$\begin{array}{l}\text { Sin posible } \\
\text { equivalente. }\end{array}$} & $\begin{array}{l}\text { Psicoticismo (no } \\
\text { cordial, competitivo, } \\
\text { carente de rectitud). }\end{array}$ & $\begin{array}{l}\text { Cordialidad. } \\
\text { Apertura a la } \\
\text { experiencia. }\end{array}$ & $\begin{array}{l}\text { Aceptabilidad. } \\
\text { Sensibilidad } \\
\text { intelectual. }\end{array}$ \\
\hline & & Conciencia. & Organización. \\
\hline
\end{tabular}

\section{La relación entre SQT y rasgos de personalidad}

Para poder hablar de la relación que existe entre el SQT y los rasgos personalidad primero se debe aclarar que los mismos estresores pueden repercutir de forma diferente dependiendo del individuo en particular del que estemos hablando, uno de los factores que están de por medio en tal situación es la personalidad. Esta última influye de manera causal en el SQT, más específicamente en el Desgaste Psíquico (El-Sahili, 2015).

En trabajos como el de Pérez-Rubio, González, \& Garcés de los Fayos (2017) los resultados planteados pueden ser calificados de inusuales ya que el rasgo llamado Cordialidad (5G) correlaciona positivamente con la Indolencia y con la apertura, así como la apertura que también correlaciona con la Baja Ilusión en el Trabajo de manera positiva. En esta misma investigación se encontró a la responsabilidad como predictivo de la baja Ilusión en el Trabajo.

Al contrario, en el estudio de Espinoza-Díaz et al. (2015) quienes trabajaron con una muestra de docentes españoles, la amabilidad, la estabilidad emocional, extroversión y la apertura a la experiencia son descritos como factores protectores del SQT, la responsabilidad es señalada también aunque en menor medida como variable protectora, al igual que Durak y Saritepeci (2019) quienes encontraron a los rasgos de personalidad; extroversión, apertura a la experiencia y estabilidad emocional en una muestra turca de docentes. Izquierdo (2015) por su parte, encontró y describió en sus resultados a la estabilidad emocional como protectora del SQT en una población mexicana de atención a clientes, Salgado y Leria (2017) por su parte, describen a la extroversión, a la responsabilidad y a la apertura a la experiencia como factores protectores del SQT aunque también como predictivos de la indolencia, estos últimos en una muestra de docentes chilenos.

En otras investigaciones en el tema, se han encontrado correlaciones entre los rasgos de Extroversión y Neuroticismo, y el Desgaste Psíquico. De Amabilidad e Indolencia de manera negativa, y con Neuroticismo de manera positiva. La Apertura a la Experiencia, la Responsabilidad, la Extraversión y la Amabilidad correlaciona positivamente con la Ilusión en el Trabajo, pero no con el Neuroticismo (Roth \& Pinto, 2015). Los rasgos de personalidad neuróticos también se asocian con el SQT para Izquierdo (2015). 
En estudios realizados con poblaciones de médicos, el rasgo de personalidad que consistentemente aparecen en los resultados es el bajo control emocional o nuroticismo (Lindeman, et al. 2017; McManus, Keeling, Paice, 2004; Prins, et al.2019). En otro estudio realizado en población coreana con estudiantes de medicina quienes trabajaron con el modelo TCI de Cloninger, Svrakic y Przybeck (1993) se encontró que la autodirección correlacionó de manera negativa con el SQT (Lee, Choi, Chae, 2017).

Un tema que se debe considerar también es la personalidad resistente, que, para Ríos, Godoy, \& Sánchez-Meca, (2011) consiste en un tipo de personalidad en la cual el individuo posee características específicas que le permiten ser "resistente" al estrés. El constructo entendido como resistencia está dividido en tres dimensiones: Compromiso que corresponde a la implicación personal en cualquier tarea; Control que corresponde a la creencia que tiene un individuo de influir en el curso de los acontecimientos; por último, Reto, este refiere a la creencia del cambio como característica inherente a la vida. Esto es importante debido a que comúnmente se aborda la relación entre personalidad resistente y SQT.

\section{Situación laboral de los docentes en México}

Las reformas educativas de los últimos años en México han impulsado cambios en las condiciones laborales de los docentes, reduciendo sus recursos percibidos e incrementando las exigencias que supone su labor, todo esto ha expuesto al docente a malestares producidos por su propio trabajo (Espinoza-Díaz, Tous-Pallarès \& Vigil-Colet, 2015). La reforma educativa (2013) emprendida por el gobierno del presidente Enrique Peña Nieto en México precarizó las condiciones laborales en cuanto a estabilidad laboral, seguridad social, prestaciones y salario de los trabajadores docentes (Gil, 2018).

Estas reformas se alinearon al marco de la globalización y el uso de nuevas tecnologías, generado cambios sociales y económicos que repercutieron en la actividad empresarial, las organizaciones y el empleo. Esto se suma a los cambios vistos en los sectores económicos como el de los servicios, sector que ha aumentado en comparación a otros sectores como la agricultura o la manufactura. En las últimas décadas México ha visto aumentos en el sector de los servicios de hasta 35\% Instituto Nacional de Estadística y Geografía, (INEGI, 2009), y en los últimos años el mismo sector ha representado alrededor del $60 \%$ de PIB nacional anual, todo esto supone un aumento en el estrés en este sector (Espinoza-Díaz et al., 2015; Figueiredo, 2014).

Ante la Reforma Educativa en México y el aumento del estrés laboral en los maestros, resulta pertinente estudiar variables implicadas en el proceso de desarrollo del SQT como la personalidad; por lo tanto, ¿cómo se expresarán las interacciones entre personalidad y SQT? El objetivo principal fue determinar el valor predictivo de los rasgos de personalidad según el modelo de los 5FM en el SQT y sus dimensiones desde el modelo del Gil-Monte. Debido a que en investigaciones anteriores los resultados no identifican a las mismas variables de personalidad se considero en los objetivos secundarios, otras variables al comparar sexos y nivel escolar impartido.

\section{Método}

El diseño fue correlacional, transversal de una sola muestra ex post facto. El tipo de muestreo fue no aleatorio por conveniencia, participaron los profesores que voluntariamente accedieron a contestar el cuestionario después de una convocatoria a todas las escuelas primarias y secundarias de la Ciudad de México. Respondieron 375 profesores de educación básica pública de 39 escuelas, seis secundarias y el resto primarias y se incluyeron los que en el momento de la aplicación se encontraran laboralmente activos, en los turnos matutino, vespertino y mixto, excluyendo aquellos que no estuvieran ejerciendo actividades dentro de la escuela, y reportaran alguna patología mental de origen no laboral. 
Del total de mujeres (280), el $16.4 \%$ de mujeres de 20 a 30 años, $35.7 \%$ de 30 a 40 años, $26.8 \%$ de 41 a 50 años y $19.3 \%$ de 51 o más años, en cuanto a los hombres $21.4 \%$. Del total de hombres (84) $21.4 \%$ de 20 a 30 años, $41.7 \%$ de 31 a $40,22.6 \%$ de 41 a 50 y $14.3 \%$ de 51 o más, el resto de los participantes (13) no contestaron.

Del Total, $48 \%$ con pareja (casados y unión libre) y $52 \%$ solteros, divorciados y viudos; 9.1\% con nivel escolar técnico, $63.5 \%$ normalista (egresado de escuelas que forma profesores de educación básica), $25.5 \%$ con licenciatura en pedagogía o área afín y $11 \%$ con posgrado. El promedio de antigüedad es de 14.3 años con $\mathrm{D} . \mathrm{E}=10.1 ; 52.3 \%$ trabaja en el turno matutino, $8.3 \%$ vespertino y el resto turno mixto. En cuanto tipo de contrato $79 \%$ es permanente y el resto interino.

\section{Instrumentos}

Cuestionario de Evaluación del Síndrome de Quemarse por el Trabajo (CESQT) validad en México en una muestra de docentes mexicanos por Gil-Monte, Unda y Sandoval, (2009) es una escala tipo Likert compuesto por 20 ítems y cuatro dimensiones, a saber de los cuales cinco corresponden a la Ilusión en el Trabajo (Alfa de .89), cuatro al Desgaste Psíquico (alfa de .89), seis a Indolencia (Alfa de .83) y cinco a Culpa (Alfa de .85), Las Alfas son de la muestra de estudio, los niveles se establecieron con base al manual de Gil-Monte (2011) y corresponden a muy bajo, bajo, alto y crítico.

Escala de los Cinco Factores Mexicanos de Personalidad (5FM): validado en población mexicana por Uribe (2002) y en población de trabajadores por Uribe y Arias, (2006) una escala tipo liker cinco opciones de respuesta y cinco dimensiones, 53 reactivos en total, de los cuales 17 corresponden a Sociabilidad expresiva (Alfa de .76), nueve de Aceptabilidad (Alfa de .73), ocho de Organización (Alfa de .46), siete de Control Emocional (Alfa de .42) y 12 de Sensibilidad Intelectual (Alfas de .65). Las Alfas corresponden a la muestra de estudio.

Cuestionario de datos sociodemográficos y sociolaborales de elaboración propia que incluye, sexo, edad, estado civil, escolaridad, antigüedad, turno y tipo de contrato.

\section{Procedimiento}

La participación de los profesores fue voluntaria, en evaluaciones colectivas realizadas en las instalaciones de los centros de trabajo (primarias y secundarias). Se les pidió a los participantes firmar un consentimiento informado en donde se aclaraba la estricta confidencialidad de la información proporcionada en los cuestionarios, así como el objetivo, y procesos subsecuentes de la investigación.

\section{Análisis de los datos}

Para el análisis de los datos obtenidos se utilizó el programa estadístico SPSS 23.0. Se aplicó un análisis de frecuencias y pruebas de tendencia central para prevalencias del SQT y sus dimensiones y de rasgos de personalidad. Se realizó una correlación bivariada de Pearson, para la relación entre Sociabilidad Expresiva, Aceptabilidad, Organización, Control Emocional Sensibilidad Intelectual e Ilusión en el Trabajo, Desgaste Psíquico, Indolencia y Culpa, esta correlación se llevó a cabo por sexo, por pertenencia a primaria o secundaria, y una regresión lineal múltiple por pasos para identificar los rasgos de personalidad predictores del SQT, así como dos regresiones lineales múltiples por pasos para distinguir sexo.

El valor de $p$ se consideró por debajo de .05 y las variables que resultaron con correlaciones significativas se integraron al modelo de regresión múltiple por pasos. 


\section{Resultados}

En cuanto a la prevalencia del SQT, el 46.1\% de los profesores presentó baja Ilusión en el Trabajo, 47.8\% de Desgaste Psíquico en un nivel alto o crítico, en Indolencia el 39\% presentó nivel alto o crítico, $31.3 \%$ tuvo Culpa en niveles altos y críticos, y 53.1\% de profesores puntuó como alto o crítico en el SQT.

En los 5FM un poco más de la mitad de los profesores tienden a presentar Sociabilidad Expresiva mientras el resto tiende a no presentarla, en el caso de Aceptabilidad 52.8\% se siente aceptado el resto rechazado, para el rasgo de Organización poco más de la mitad tiende a ser organizado el resto desorganizado, 53.3\% tiende al Control Emocional, el resto a no presentar el rasgo. Y en Sensibilidad Intelectual 55.7\% tiende a no presentar el rasgo, el resto a presentarlo.

Como se puede observar en la Tabla 2, en el resultado del análisis de correlación las relaciones más altas se encontraron de forma negativa entre Sociabilidad Expresiva y Desgaste Psíquico ( $\left.\mathrm{r}=-.462^{* *}\right)$, Sociabilidad Expresiva y SQT ( $\left.\mathrm{r}=-.441^{* *}\right)$, Aceptabilidad y SQT ( $\mathrm{r}=-$ $.419 * *)$ Sociabilidad Expresiva y Culpa $(\mathrm{r}=-.392 * *)$, y Aceptabilidad y Degaste Psíquico ( $\mathrm{r}=-$ .380**). La única correlación no significativa fue Control Emocional e Ilusión en el Trabajo $(\mathrm{r}=.087)$.

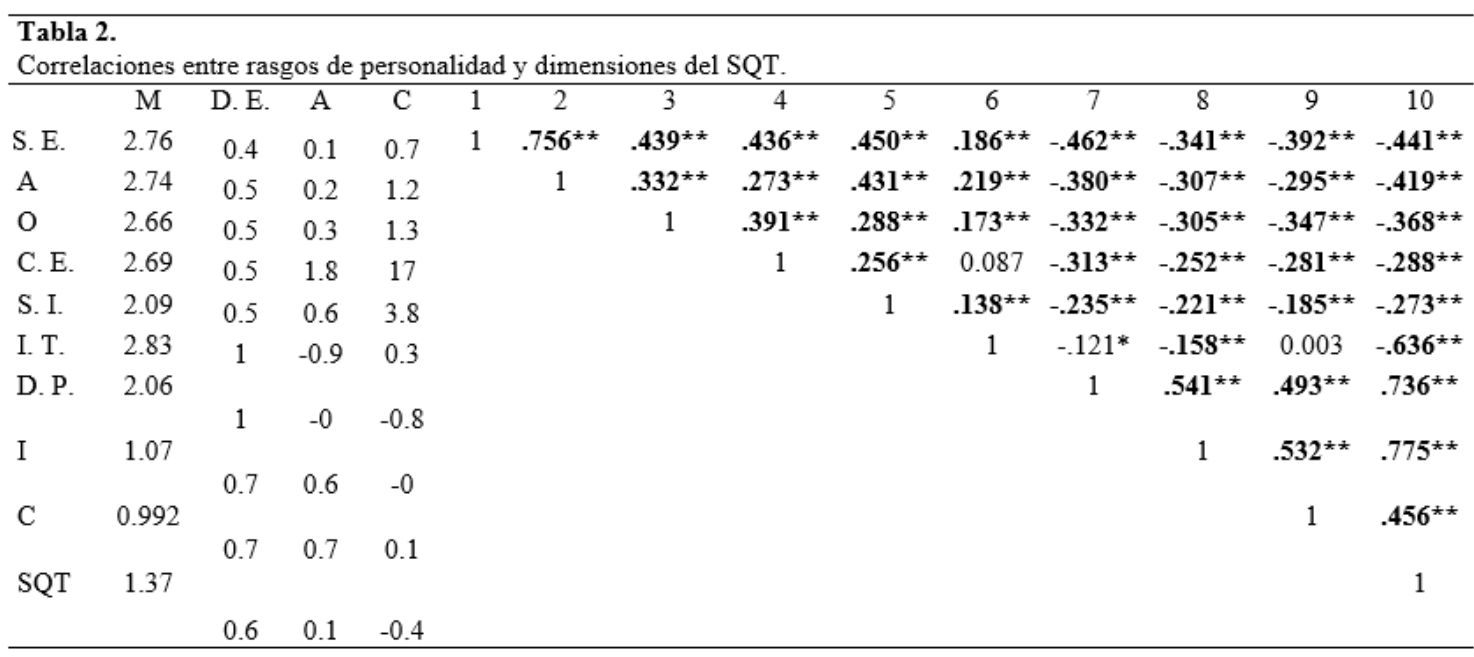




\section{Tabla 3.}

Regresiones lineales entre rasgos de personalidad y el Síndrome de Quemarse por el Trabajo

\begin{tabular}{|c|c|c|c|c|c|c|c|c|c|}
\hline Modelos & $\mathrm{F}$ & R2 & $\Delta \mathrm{R} 2$ & B & $\begin{array}{c}\text { Error } \\
\text { estándar }\end{array}$ & $\beta$ & $\mathrm{p}$ & $1-\beta$ & $f$ \\
\hline & \multicolumn{9}{|c|}{ Ilusión en el trabajo } \\
\hline Paso 1 & $\begin{array}{c}15.94 \\
(1,350)\end{array}$ & .044 & .041 & & & & .001 & .75 & .046 \\
\hline Aceptabilidad & & & & .361 & .253 & .209 & & & \\
\hline Paso 2 & $\begin{array}{c}10.48 \\
(2,349)\end{array}$ & .057 & .051 & & & & .028 & .98 & .060 \\
\hline Aceptabilidad & & & & .291 & .095 & .168 & .002 & & \\
\hline \multirow[t]{2}{*}{ Organización } & & & & .238 & .108 & .121 & .028 & & \\
\hline & \multicolumn{9}{|c|}{ Desgaste Psíquico } \\
\hline Pasol & $\begin{array}{c}94.33 \\
(1,351)\end{array}$ & .212 & .210 & & & & .001 & 1 & .269 \\
\hline Sociabilidad expresiva & & & & -1.011 & .104 & -.460 & .001 & & \\
\hline Paso 2 & $\begin{array}{l}55.560 \\
(2,350)\end{array}$ & .241 & .237 & & & & .001 & 1 & .317 \\
\hline Sociabilidad Expresiva & & & & -.824 & .114 & -.375 & .001 & & \\
\hline \multirow[t]{2}{*}{ Organización } & & & & -.396 & .108 & -.191 & .001 & & \\
\hline & \multicolumn{9}{|c|}{ Indolencia } \\
\hline Paso 1 & $\begin{array}{l}44.918 \\
(1,349)\end{array}$ & .114 & .111 & & & & .001 & .99 & .128 \\
\hline Sociabilidad expresiva & & & & -.514 & .083 & -.338 & .001 & & \\
\hline Paso 2 & $\begin{array}{l}16.102 \\
(2,348)\end{array}$ & .150 & .145 & & & & .001 & .99 & .176 \\
\hline Sociabilidad expresiva & & & & -.399 & .091 & -.243 & .001 & & \\
\hline \multirow[t]{2}{*}{ Organización } & & & & -.328 & .085 & -.212 & .001 & & \\
\hline & \multicolumn{9}{|c|}{ Culpa } \\
\hline Paso 1 & $\begin{array}{c}61.487 \\
(1,348)\end{array}$ & .150 & .148 & & & & .001 & .99 & .176 \\
\hline Sociabilidad expresiva & & & & -.656 & .084 & -.388 & .001 & & \\
\hline Paso 2 & $\begin{array}{l}40.434 \\
(2,347)\end{array}$ & .189 & .184 & & & & .001 & 1 & .233 \\
\hline Sociabilidad expresiva & & & & -.492 & .091 & -.290 & .001 & & \\
\hline \multirow[t]{2}{*}{ Organización } & & & & -.349 & .086 & -.220 & .001 & & \\
\hline & \multicolumn{9}{|c|}{ SQT } \\
\hline Paso 1 & $\begin{array}{l}84.360 \\
(1,347)\end{array}$ & .196 & .193 & & & & .001 & 1 & .243 \\
\hline Sociabilidad expresiva & & & & -.613 & .067 & -.442 & .001 & & \\
\hline Paso 2 & $\begin{array}{l}55.045 \\
(2,346)\end{array}$ & .241 & .237 & & & & & 1 & .317 \\
\hline Sociabilidad expresiva & & & & -.465 & .072 & -.336 & .001 & & \\
\hline Organización & & & & -.310 & .068 & -.239 & .001 & & \\
\hline Paso 3 & $\begin{array}{l}39.892 \\
(3,345)\end{array}$ & .258 & .251 & & & & .006 & 1 & .347 \\
\hline Sociabilidad expresiva & & & & -.256 & .105 & -.185 & .015 & & \\
\hline Organización & & & & -.312 & .067 & -.241 & .001 & & \\
\hline Aceptabilidad & & & & -.226 & .082 & -.197 & .006 & & \\
\hline
\end{tabular}

El análisis de regresión lineal múltiple por pasos en el que se integraron todos los rasgos de personalidad y las dimensiones del síndrome, se señala como predictores de Ilusión en el Trabajo $\{\mathrm{F}=10.4,(\mathrm{gl}=2 / 349)\}^{* *}$ a la Aceptabilidad $\left(\beta=.209^{* *}\right)$ en el primer paso, y Aceptabilidad $\left(\beta=.168^{* *}\right)$ con Organización $\left(\beta=.121^{*}\right)$ que se integraron en el segundo, la potencia estadística es aceptable y el tamaño del efecto es bajo, el total de la varianza explicada del modelo es $5.7 \%$; para Desgaste psíquico $\{\mathrm{F}=55.5,(\mathrm{gl}=2 / 350)\}^{* *}$, Sociabilidad Expresiva (en primer paso $\beta=$ - 
$.460 * *)$ y el segundo Sociabilidad Expresiva $\left(\beta=-.375^{* *}\right)$ y Organización $\left(\beta=-.191^{* *}\right)$, la potencia estadística es buena y el tamaño del efecto medio, el total de la varianza explicada del modelo es $24.1 \%$; del mismo modo en la Indolencia $\{\mathrm{F}=16.1,(\mathrm{gl}=2,348)\}^{* *}$, se integró en el primero paso a la Sociabilidad Expresiva ( $\left.\beta=-.338^{* *}\right)$ y en el segundo Sociabilidad Expresiva $(\beta=-.243 * *)$ y Organización $(\beta=-.212 * *)$ la potencia estadística es adecuada y el tamaño del efecto es bajo, el total de la varianza explicada del modelo es $15 \%$; para Culpa $\{\mathrm{F}=40.4,(\mathrm{gl}=2,347)\}^{* *}$ Sociabilidad Expresiva $\left(\beta=-.388^{* *}\right)$ en el primer paso, y Sociabilidad Expresiva $\left(\beta=-.290^{* *}\right)$ con Organización $\left(\beta=-.220^{* *}\right)$ en el segundo, la potencia estadística es buena y el tamaño del efecto es medio, el total de la varianza explicada del modelo es $18.9 \%$. Por último para el SQT $\{\mathrm{F}=39.8$, $(\mathrm{gl}=2,347)\}^{* *}$, Sociabilidad Expresiva $\left(\beta=-.442\right.$ en el primer paso), Sociabilidad $\left(\beta=-.336^{* *}\right)$ con Organización $(\beta=.239 * *)$ (integrados en el segundo paso), Sociabilidad $\left(\beta=-.185^{*}\right)$, Organización $\left(\beta=-.241^{* *}\right)$ y Aceptabilidad $(\beta=-.197 * *)$ (integrados en el tercer paso), la potencia estadística es buena y el tamaño del efecto es medio, el total de la varianza explicada del modelo es $25.1 \%$ (ver Tabla 3 ).

\section{Discusión}

El objetivo principal fue determinar el valor predictivo de los rasgos de personalidad según el modelo de los 5FM en el SQT y sus dimensiones desde el modelo del Gil-Monte.

La prevalencia realizada en población mexicana por Unda, Sandoval, \& Gil-Monte (2008) hace diez años presentó resultados menores a los del presente estudio, la baja o muy baja Ilusión por el Trabajo pasó de estar en un 3\% a un $46.1 \%$, el Desgaste Psíquico paso de 37.4\% en alto o critico a $47.8 \%$, lo mismo con la Culpa que pasó de $9.70 \%$ a 31.3\%, y SQT alto o crítico que pasó de $35.5 \%$ al $53.1 \%$.

Estos resultados pueden explicarse por la aprobación de la Reforma Educativa del 2013 en México, que planteó una evaluación que fue calificada por los profesores como laboral, pues al no aprobar la evaluación, los profesores podrían perder su trabajo, independientemente de su contrato permanente o de su antigüedad, lo anterior se acompañó de una mayor supervisión de su trabajo y controles mas estrictos en su desempeño, ampliación de las jornadas laborales, incremento de actividades administrativas entre otros, (INEE, 2015; Unda, 2018) lo anterior contribuyó a un incremento del estrés laboral y a mayor presencia del Burnout y diversos trastornos (Palacios, Morales \& Estrella, 2018; Rodríguez, Tovalín, Gil- Monte, Salvador \& Acle, 2018).

En cuanto al objetivo la correlación realizada todas las asociaciones significativas fueron negativas a excepción de aquellas que incluyen a la Ilusión por el Trabajo, los rasgos de personalidad que obtuvieron los valores más altos al correlacionar con el SQT y sus dimensiones fueron Sociabilidad Expresiva y Aceptabilidad, esto es esperado de acuerdo a la teoría, puesto que los individuos con características extrovertidas o comparables con la personalidad resistente tiene más posibilidades de anteponerse al Síndrome.

Las investigaciones realizadas con anterioridad han encontrado una relación existente entre la personalidad y el SQT como lo plantea la teoría, ya sea estudiándola a través de constructos como la personalidad resistente, de la personalidad eficaz, o simplemente a través de la teoría de los rasgos, haciendo pertinente que los resultados en un estudio como el presente incluyan regresiones como las llevadas a cabo (Espinoza-Díaz et al., 2015; Izquierdo, 2015; Pérez-Rubio et al., 2017; Ríos et al., 2011; Salgado \& Leria, 2017).

En el modelo de regresión de la muestra general, los rasgos señalados como predictivos de la Ilusión en el Trabajo son Aceptabilidad $\left(\beta=.209^{* *}\right)$ y Organización $\left(\beta=.121^{*}\right)$, el papel del primer rasgo podría explicarse mediante el sentimiento de aceptación que representa, el cual abona a la percepción que tiene el docente de que el aporta cosas positivas a su trabajo. La 
Organización por su parte fomenta conductas que puedes ser calificada como positivas a través de los estilos de trabajo, lo cual aumenta la Ilusión Alvares \& Obiols (2009).

En el caso del Desgaste Psíquico los rasgos de personalidad señalados como predictivos fueron Sociabilidad Expresiva $(\beta=-.460 * *)$ y Organización $(\beta=-.191 * *)$, el caso específico de la Sociabilidad Expresiva nos permite sumar a la hipótesis que señala a las redes de apoyo como variables protectoras, algo esperado teóricamente puesto que la Sociabilidad expresiva supone una representación de cómo el individuo interactúa con otros, el Desgaste Psíquico por su parte, representa el componente emocional del Síndrome y representa parte importante de la afectación del mismo. Por otro lado, y con respecto a la Organización los profesores que presentan altos puntajes podrían encontrar en esta situación una herramienta, que en este caso sería utilizado para afrontar situaciones estresantes.

Para la Indolencia, los rasgos identificados como predictivos son Sociabilidad Expresiva $\left(\beta=-.338^{* *}\right)$ y Organización $\left(\beta=-.212^{* *}\right)$. Con base en esto se puede pensar que aquellos profesores que presentan altos puntajes en Sociabilidad Expresiva, tienen también nexo con la comunidad estudiantil lo cual podría inhibir las actitudes negativas o indolentes ante los estudiantes. Por otro lado, la Organización puede ser explicada como una herramienta que podría permitirle al profesor tomar decisiones más sistemáticas al tratar a los alumnos, al respecto Alvares \& Soler, (2009) señalan el proceso que supone la toma de decisiones en un contexto profesional implica un proceso cognitivo en el cual las habilidades para organizar información tienen un papel importante. Lo mismo podría ocurrir al contrario cuando la Organización dificulte la toma de decisiones del profesor al respecto Cobarrubias \& Piña (2004) señalan la importancia de ser flexible en la profesión docente.

Para la culpa los rasgos encontrados como predictivos fueron Sociabilidad Expresiva $(\beta=-$ $.338 * *)$ y Organización $(\beta=-.220 * *)$. La correlación esperada teóricamente entre Control Emocional y la Culpa no resultó significativa. Otra hipótesis orientada por los resultados es que la Culpa tiene un nexo teórico con las conductas indolentes, es pues producto de estas lo cual podría explicar la presencia de tales rasgos en el modelo de regresión. Otro argumento relacionado con la Organización tiene que ver con las capacidades que tiene un docente organizado, ya que este tendría más herramientas con las cuales determinar si las sanciones o represalias determinadas por él hacia el alumnado corresponden a su criterio moral.

El modelo de regresión del SQT señala como predictores a la Sociabilidad Expresiva $(\beta=-$ $.442 * *)$, Organización $\left(\beta=-.239^{* *}\right)$ y Aceptabilidad $(\beta=-.197 * *)$. Los tres rasgos ya mencionadas y explicados en las dimensiones anteriores del Síndrome también pueden ser explicadas mediante la muestra, y no solo mediante sí mismas, estos rasgos son características valoradas en la docencia, el liderazgo como Sociabilidad Expresiva, una figura de autoridad que implica la Organización y un papel fundamental en la comunidad y su desarrollo que representaría la Aceptación.

Los resultados de este estudio difieren con los de Pérez-Rubio et al. (2017) con hallazgos en otras profesiones, por ejemplo, jugadores profesionales de juegos electrónicos, encontraron correlaciones positivas entre Cordialidad y SQT, así como entre Organización y SQT, otro estudio que difiere es el de Roth \& Pinto (2015) quienes encontraron las relaciones Estabilidad Emocional con Desgaste psíquico e indolencia en una muestra de enfermeras. Tambien las investigaciones realizadas en poblaciones de médicos presentaron resultados diferentes, señalando al neuroticismo como un rasgo con papel en el desarrollo del SQT (Lindeman, et al. 2017; McManus, Keeling, Paice, 2004; Prins, et al.2019).

A diferencia de la situación anterior, Espinoza-Díaz et al. (2015), trabajando con una muestra de profesores de educación básica al igual que el presente estudio, encontraron a la Conciencia y a la Extroversión como protectores esta última con más fuerza que la primera, como se señala en la Tabla 1 estos resultados son semejantes a los de esta investigación. Además, 
encontraron a la Estabilidad Emocional o bajo Neocriticismo, la Amabilidad y la Apertura a la Experiencia como protectores del SQT. Otros autores que coinciden con estos últimos planteamientos son Salgado \& Leria (2017) quienes encontraron en una muestra de enfermeras asociación entre el SQT y el Neuroticismo, y al igual que en los resultados de nuestro estudio detectaron a la Extroversión o Sociabilidad Expresiva y a la Organización como protectores del SQT.

La personalidad resistente en la definición de Ríos (2011) está caracterizada por aspectos del individuo que puede de alguna forma equivaler al rasgo que llamamos alta Sociabilidad Expresiva, un ejemplo de esto es que los individuos cuya personalidad es calificada de resistente, creen que el cambio es algo propio de la vida lo cual encaja con la caracterización osada y expectante del Individuo que puntúa como alto en el rasgo de personalidad Sociabilidad Expresiva. Otra característica de la personalidad resistente es el compromiso, el cual coincide también con los resultados de este estudio, ya que una persona comprometida bien puede ser organizada.

Es importante resaltar que aquellas investigaciones con resultados semejantes a los de este estudio tienen muestras semejantes también, lo que permite suponer que la relación entre SQT y rasgos de personalidad varía según otras variables como la tarea desempeñada, sumando a la explicación multicausal del SQT.

En resumen, podemos decir que los rasgos de personalidad tienen un papel mediador en la aparición del SQT, específicamente los individuos que presentan baja Sociabilidad Expresiva, baja Organización y baja Aceptabilidad son más propensos a presentar SQT.

Sin embargo, la personalidad no puede asumirse como el factor determinante en un fenómeno de múltiples determinaciones. Los resultados solo pueden abonar a la explicación multicausal del síndrome.

Una primera limitación de este estudio fue no incluir los factores de riesgo psicosocial de origen laboral para analizar el papel mediador de los rasgos de personalidad en el desarrollo del síndrome. Se sugiere para estudios posteriores incluir variables como lo son las malas relaciones interpersonales, falta de apoyo social y ambigüedad de rol.

Una segunda limitación importante fue las bajas puntuaciones de la prueba de fiabilidad Alfa de Cronbach en algunas dimensiones de la escala de personalidad de los 5FM. La decisión de utilizarla fue que los autores Uribe, (2002); Uribe y Arias, (2005); y Uribe, Contreras, Sánchez, y García, (2008) reportaron Alfas adecuadas en diferentes poblaciones de trabajadores mexicanos. Dichos estudios realzan la necesidad de utilizar instrumentos para evaluar la personalidad propia de cada cultura, por lo tanto, para próximas investigaciones será necesario adecuar el modelo propuesto a la población estudiada o diseñar una nueva propuesta propia para trabajadores docentes.

Una tercera limitación del estudio fue el tipo de muestreo que no fue aleatorio, lo que impide generalizar los resultados a los profesores de educación básica mexicanos.

Los hallazgos del estudio en relación con la prevalencia sugieren una veta para seguir profundizando en relación con los impactos a la salud que trae los cambios legislativos en materia educativa en México impulsados en el 2013, y en el caso de la relación entre los rasgos de personalidad y el SQT se recomienda profundizar en las diferencias de cómo se presenta esta relación según el sexo y según la actividad a desempeñar.

\section{Referencias}


Aldrete, M., Pando, M., Aranda, C., \& Balcázar, N. (2003). Síndrome de Burnout en maestros de educación básica nivel primario de Guadalajara. Revista Investigación en salud, 5(1), 110

Recuperado de http://www.redalyc.org/articulo.oa?id=14200103

Ayuso, J., \& Guillen, C. (2007). Burnout y Mobbing en enseñanza secundaria. Revista Complutense de Educación, 19(1), 157-173. Recuperado de https://revistas.ucm.es/index.php/RCED/article/view/RCED0808120157A

Cloninger, S. (2003). Teorías de la personalidad. $3^{\mathrm{a}}$ ed, Ciudad de México: Pearson Educación

Covarrubias, P., \& Piña, M. (2004). La interacción maestro-alumno y su relación con el aprendizaje. Revista Latinoamericana de Estudios Educativos (México), 34(1), 47-84.

Barbosa, L., Muñoz, M., Rueda, P., \& Suárez, K. (2009). Síndrome de SQT y estrategias de afrontamiento en docentes universitarios. Revista Iberoamericana de Psicología Ciencia y Tecnología, 2(1), 21-30 https://revistas.iberoamericana.edu.co/index.php/ripsicologia/article/view/174

Díaz, F., \& Gómez, I. (2016). La investigación sobre el Síndrome de Burnout en Latinoamérica entre 2000 y el 2010. Psicología desde el Caribe, 1(33): 113-131 http://www.scielo.org.co/pdf/psdc/v33n1/v33n1a09.pdf

INEE. (2015). Los docentes en México. Recuperado de https://www.inee.edu.mx/images/stories/2015/informe/Los_docentes_en_Mexico._Infor me_2015_1.pdf

El-Sahili, L. (2015). Áreas que envuelven al docente y gestan su burnout. En: Burnout. Consecuencias y soluciones. $1^{\mathrm{a}}$ ed. Ciudad de México: Manual Moderno, p. 69-84.

Espinoza-Díaz, I., Tous-Pallarès, J., \& Vigil-Colet, A. (2015). Efecto del Clima Psicosocial del Grupo y de la Personalidad en el Síndrome de Quemado en el Trabajo de los docentes. Anales de Psicología, 31(2), 651-657. Recuperado de $\mathrm{http} / / /$ scielo.isciii.es/scielo.php?script=sci_arttext\&pid=S0212-97282015000200029

Figueiredo, H. (2014). Análisis de la influencia de los sentimientos de culpa en el proceso de desarrollo del Síndrome de Quemarse por el Trabajo (SQT) y sus consecuencias. Un estudio transcultural (1era. ed.) Valencia: Universidad de Valencia: España. Recuperado de http://roderic.uv.es/handle/10550/37718

Figueiredo-Ferraz, H., Grau-Alberola, E., \& Gil-Monte, P. (2016). Influencia de los valores y la culpa en el síndrome de burnout. Psicologia do Trabalho e das Organizações. 85-95. Recuperado de https://www.uv.es/unipsico/pdf/Publicaciones/Capitulos/01_SQT/2016_Braga.pdf

Gil-Monte, P. R., Unda S., y Sandoval J. I. (2009). Validez factorial del "Cuestionario para la Evaluación del Síndrome de Quemarse por el Trabajo" (CESQT) en una muestra de maestros mexicanos. Salud Mental, 32(3), 205-214 Recuperado de http://www.scielo.org.mx/pdf/sm/v32n3/v32n3a4.pdf

Gil, M. (2018). La reforma educativa, facturas estructurales. Revista Mexicana de Investigación Educativa, 23(76), 303-321. Recuperado de http://www.comie.org.mx/documentos/rmie/v23/n076/pdf/76012.pdf

Gil-Monte, P. (2005). Síndrome de quemarse por el trabajo (burnout) $1^{\mathrm{a}}$ ed, Madrid: Pirámide.

Gil-Monte, P. (2007). El síndrome de quemarse por el trabajo (burnout) una perspectiva histórica. En Gil-Monte PR, Moreno-Jiménez B, editores. El síndrome de quemarse por el trabajo (burnout) (1 era ed.) Madrid: Pirámide, p. 21-42.

Gil-Monte, P. (2011). Cuestionario para la evaluación del Síndrome de Quemarse por el Trabajo. $1^{\mathrm{a}}$ ed, Madrid: Tea Ediciones.

González, M. Á., \& Soler, M. O. (2009). El proceso de toma de decisiones profesionales a través del coaching. Electronic Journal of Research in Educational Psychology, 7(2), 877-900.

Holland, J. (1966). The psychology of Vocational Choise. A Theory of personality types and model environments. Waltham, MA: Blaisdell.

Instituto Nacional de Estadística y Geografía, INEGI. (2009). Economía de México. Recuperado de:

http://cuentame.inegi.org.mx/economia/default.aspx?tema=E 
Izquierdo, M. (2015). Síndrome de Quemarse por el trabajo (SQT) en los empleados de atención a clientes de telefonía móvil (Metepec, 2015). Diagnóstico como base para propuestas de afrontamiento y prevención (1era ed.). Universidad Autónoma del Estado de México. Recuperado de http://ri.uaemex.mx/bitstream/handle/20.500.11799/67832/TTG_Izquierdo.pdf?sequence $=3$

Llanque, A., (2014), Rasgos de Personalidad y Síndrome de Burnout en personal de Salud de la Unidad de quemados y cirugía plástica del "Hospital de Clínicas" de la Ciudad de la Paz, Lecturas en Psicología, (115) 12-93.

Maslach, C., \& Jackson, S. E. (1981). The measurement of experienced burnout. Journal of organizational behavior, 2(2), 99-113.

Maslach, C., \& Leiter, M. P. (2016). Understanding the burnout experience: recent research and its implications for psychiatry. World Psychiatry, 15(2), 103-111.

Moreno-Jiménez, B., Gonzalez, J., \& Garrosa, E. (2001). Desgaste profesional (Burnout), Personalidad y Salud percibida. En; Buendia J, Ramos F. Empleo editores, estrés y Salud. 1 a Ed. España: Pirámide, p. 59-83.

Ortiz, M., Castelvi, M., Espinoza, L., Guerrero, R., Lienqueo, P., Parra, R., y Villagra, E. (2012). Tipos de personalidad y síndrome de burnout en educadoras de párvulos en Chile. Universitas Psychologica, 11(1) 229-239.

Palacios, R., Morales, E., \& Estrella, G. (2018). Sobrecarga laboral, tensiones dentro y fuera del aula: docentes enfermos, estresados. Intercambio, 12, 17-21. Recuperado de http://ideanetwork.ca/wp-content/uploads/2018/04/4-Sobrecarga-laboral.pdf

Pérez-Rubio, C., González, J., \& Garcés de los Fayos, E. (2017). Personalidad y burnout en jugadores profesionales de e-sports. Cuadernos de Psicología del Deporte, 17 (1), 41-50. Recuperado de http://revistas.um.es/cpd/article/view/291901

Pines, A., \& Keinan, G. (2005). Stress and Burnout: The significant difference. Personality and individual differences, 39(2005), 625-635. Recuperado de http://isiarticles.com/bundles/Article/pre/pdf/58875.pdf

Rabasa, B., Figueiredo-Ferraz, H., Gil-Monte, P., \& Llorca-Pellicer, M. (2016). El papel de la culpa en la relación entre el síndrome de quemarse por el trabajo y la inclinación al absentismo de profesores de Enseñanza Secundaria. Revista de Psicodidáctica, 21(1), 103119.

Recuperado

de http://www.ehu.eus/ojs/index.php/psicodidactica/article/viewFile/13076/13429

Ríos, M., Godoy, C., \& Sánchez-Meca, J. (2011). Síndrome de quemarse por el trabajo, personalidad resistente y malestar psicológico en personal de enfermería. Servicio de Publicaciones de la Universidad de Murcia, 27(1), 71-79. Recuperado de http://www.redalyc.org/html/167/16717018009/

Roth, E., \& Pinto, B. (2015). Síndrome de Burnout, Personalidad y Satisfacción Laboral en Enfermeras de la Ciudad de La Paz. Ajayu, 8(2), 65-102. Recuperado de http://www.ucb.edu.bo/publicaciones/Ajayu/v8n2/v8n2a4.pdf

Salgado, J., \& Leria, F. (2017). Síndrome de Burnout y calidad de vida profesional percibida según estilos de personalidad en profesores de educación primaria. Revista CES, 11(1), 6989. Recuperado de https://dialnet.unirioja.es/servlet/articulo?codigo=6337819

Silva, M., García, R., González, M., Ratto, A. (2015). Prevalencia del Síndrome de Quemarse por el Trabajo y variables sociodemográficas en un grupo de maestras de Montevideo. Ciencias $\begin{array}{llll}\text { Psicológicas, } & 9(1): & \text { 55-62. } & \text { Recuperado }\end{array}$ http://www.scielo.edu.uy/pdf/cp/v9n1/v9n1a06.pdf

Unda, S., Sandoval, J., \& Gil-Monte, R. (2008). Prevalencia del Síndrome de Quemarse por el Trabajo (SQT) (Burnout) en maestros mexicanos. Información Psicológica. 53-63. Recuperado http://www.informaciopsicologica.info/OJSmottif/index.php/leonardo/article/download/2 $64 / 217$

Uribe, J. (2002). Desarrollo y validación de la escala de los Cinco Factores Mexicanos de Personalidad (5FM): Selección de personal y éxito profesional. Revista de la Universidad 
Cristóbal Colón. 15(5), 105-128. Recuperado de http://scielo.isciii.es/scielo.php?script=sci_nlinks\&ref=4671367\&pid=S1576$5962200800010000400070 \& \operatorname{lng}=\mathrm{es}$

Uribe, J. \& Arias, F. (2005). Un estudio de la confiabilidad y la validez del 5FM: Cinco Factores de la Personalidad en la Cultura Mexicana. Revista Interamericana de Psicología ocupacional. 24(1): 16-30.

Uribe, J., Contreras, F., Sánchez, O., García, A. (2008). Los Cinco Grandes y maquiavelismo en trabajadores mexicanos: un estudio de personalidad y manipulación. Revista de Psicología del Trabajo $y$ de las Organizaciones. 24(1), 61-79. Recuperado de $\mathrm{http} / / /$ scielo.isciii.es/pdf/rpto/v24n1/v24n1a04.pdf

Vicent, M., Inglés, C., Gonzálvez, C., Sanmarin, R., \& García-Fernández, J. (2016). Perfeccionismo socialmente prescrito y los cinco grandes rasgos de la personalidad en niños españoles. Eurpean Journal of Investigation in Health. 6(2), 107-118. Recuperado dehttps://rua.ua.es/dspace/bitstream/10045/57295/1/2016_Vicent_etal_EJIHPE.pdf

\section{Agradecimiento}

Agradecemos al proyecto PAPIIT IN306118 por el apoyo brindado a esta investigación. 